\title{
DIE BANKENVERSTAATLICHUNG IN INDIEN
}

\author{
Von BRUn-OtTo BRYDE
}

\section{Politische Hintergründe}

Am 19. Juli 1969 wurden die 14 größten Banken Indiens durch Ordinance des Präsidenten nach Art. 123 der Verfassung verstaatlicht. Ministerpräsidentin Frau Indira Ghandi begründete diesen Schritt noch am gleichen Abend in einer Radioansprache ${ }^{1}$. Sie sagte u. a., die Kontrolle der „Kommandohöhen“ der Wirtschaft sei vor allem in einem armen Land notwendig, in dem es außerordentlich schwierig sei, Hilfsquellen für die Entwicklung zu mobilisieren und Ungleichheiten zwischen verschiedenen Gruppen und Regionen zu beseitigen. Die Verstaatlichung werde die Kontrolle durch wenige beseitigen und gewährleisten, daß ausreichender Kredit für bisher vernachlässigte Bereiche wie die Landwirtschaft, kleine Industriebetriebe und den Export zur Verfügung stehe. Den Verdacht, man stehe am Beginn einer Welle von Nationalisierungen, wies sie zurück.

Die Rede der Ministerpräsidentin konnte die wenigsten Beobachter überzeugen, daß die angeführten Argumente der eigentliche Beweggrund für eine Bankenverstaatlichung zu diesem Zeitpunkt waren². Die Nationalisierung der von wenigen reichen Familien kontrollierten Banken war in der Kongreßpartei zwar schon lange in der Diskussion. Noch das Zehnpunkteprogramm der Partei von $1967^{3}$ hatte jedoch statt der Verstaatlichung lediglich die gesellschaftliche Kontrolle („social control“) der Banken gefordert4. Dieser Programmpunkt war durch den Banking Laws (Amendment) Act vom Dezember $1968^{5}$ verwirklicht worden, durch den eine weitgehende Regierungskontrolle vor allem der Kreditvergabepolitik der Banken gesichert wurde. Im Juli 1969 brachte die Ministerpräsidentin das Thema der Bankenverstaatlichung auf der Sitzung des All-India Congress Committees (A.I.C.C.) erneut ins Gespräch, aber wiederum nur als mögliche Alternative zur "social control“6. Seit dem Inkrafttreten der "social control“-Gesetzgebung? war zu wenig Zeit vergangen, als daß ihr Scheitern sich bereits hätte feststellen lassen. Überdies war sie am 14. Dezember 1968 im Unterhaus (Lok Sabha) von Finanzminister Desai mit den gleichen Argumenten begründet worden (Beseitigung der Kontrolle durch wenige, Kredit für bisher vernachlässigte Wirtschaftssektoren) ${ }^{8}$, mit denen die Ministerpräsidentin nunmehr die Verstaatlichung verteidigte.

Es ist daher nicht verwunderlich, daß viele Beobachter sofort die Vermutung äußerten, ihre Entscheidung habe mehr innen- und parteipolitische als wirtschaftspolitische Motive ${ }^{9}$. In der sich verschärfenden Auseinandersetzung $\mathrm{zwischen} \mathrm{der}$ Ministerpräsidentin und der Kongreßführung („Syndikat“), die schließlich zur Spal-

\footnotetext{
1 Auszüge bei Keesing's 1969, S. 23559. Vgl. auch Herald Tribune vom 21. 7. 1969. Neue Zürcher Zeitung (NZZ) vom 21. 7. 69

2 Vgl. NZZ vom 21. 7. 1969, weitere Nachweise bei Keesing's 1969, S. 23559.

3 Vgl. dazu Kochanek, The Congress Party of India, Princeton 1968, S. $417 \mathrm{ff}$.

4 Während für das Versicherungswesen die Verstaatlichung verlangt wurde vgi. Kochanek, a. a. O., S. 417.

5 Vgl. Keesing's 1969, S. 2355.

6 Vgl. Keesing's 1969, S. $2357 \mathrm{f}$

7 Banking Laws (Amendment) Act, vgl. Anm. 6.

8 Auszüge seiner Rede bei Keesing's 1969, S. 23557.

9 Vgl. z. B. NZZ vom 21. 7. 1969, Nationalisierung der indischen Großbanken - Ein Mittel im Machtkampf um die Kongreßführung; weitere Nachweise bei Keesing's 1969, S. 23559.
} 
tung der Partei führte ${ }^{10}$, bot sich die Bankenverstaatlichung aus verschiedenen Gründen als Kampfmittel an. Die Gegner Frau Ghandis im "Syndikat" waren zum Teil öffentlich auf eine Bankenverstaatlichung festgelegt (Kamaraj und Chavan), zum anderen Teil als Gegner einer solchen Maßnahme bekannt (Desai, Nijalingappa, Patil), und mußten daher in Schwierigkeiten kommen. Zum anderen lag der Ministerpräsidentin daran, ihrem Machtkampf mit dem "Syndikat" eine ideologische Färbung zu geben, und so den progressiven Flügel der Partei auf ihre Seite zu bringen. Schließlich konnte Frau Ghandi hoffen, sich für den Fall eines Auseinanderbrechens der Kongreßpartei als Führerin einer Koalition der Linksparteien ${ }^{11}$ zu empfehlen. Eine möglicherweise entscheidende Rolle dürfte in den Überlegungen der Ministerpräsidentin jedoch die Auseinandersetzung mit ihrem Hauptgegner, Finanzminister Desai, gespielt haben. Diesen hatte sie am 16. Juli 1969 als Finanzminister entlassen ${ }^{12}$. In einem Brief ${ }^{13}$ begründete sie den Schritt mit seinen Vorbehalten gegenüber ihrer Wirtschaftspolitik. Die Fairness gebiete es ihr, ihn der Last, diese Politik als Finanzminister durchzusetzen, zu entheben und das Amt selbst zu übernehmen. Da Desai vorher alles getan hatte, diesen Vorwand zu vermeiden (er hatte z. B. die Resolution des A.I.C.C. von Bangalore, die die Wirtschaftspolitik Frau Ghandis befürwortete, uneingeschränkt unterstützt), liegt die Vermutung nahe, daß die Ministerpräsidentin mit dem radikalen Schritt der Bankenverstaatlichung, deren Ablehnung durch Desai bekannt war, beweisen wollte, daß der Finanzminister ihre Politik nicht unterstützen könne. Desai selbst hat diesen Grund als entscheidendes - wenn nicht einziges - Motiv für die Bankenverstaatlichung bezeichnet ${ }^{14}$.

Falls die Motive für die Bankenverstaatlichung tatsächlich die genannten waren, muß man die Maßnahme als erfolgreich bezeichnen. Das "Syndikat" konnte wegen der divergierenden Meinungen in seiner Mitte in diesem Punkt nicht den Kampf aufnehmen, und die Ministerpräsidentin gewann die Unterstützung von Linkskreisen innerhalb und außerhalb der Kongreßpartei ${ }^{15}$.

\section{Die Verstaatlichungsmaßnahmen}

Durch die Ordinance vom 19. Juli 196916 wurden insgesamt 14 Banken verstaatlich $t^{17}$. Von der Nationalisierung ausgenommen wurden Banken mit weniger als 500 Mill. Rs. Einlagen und ausländische Banken.

Die Verstaatlichung wurde in der Weise vorgenommen, daß 14 neue staatliche Banken gegründet wurden, auf die das gesamte Aktiv- und Passivvermögen der enteigneten Gesellschaften überging ${ }^{18}$. Die Leitung der neuen Banken sollte bei einem von der Regierung ernannten „custodian“ liegen. Dieses Amt übernahmen zunächst die Vorstandsvorsitzenden der enteigneten Gesellschaften ${ }^{19}$. Alle Ange-

$10 \mathrm{Vgl}$. Stiebler, Klaus, Die Spaltung der Kongreßpartei und ihre Folgen, in: Internationales Asienforum 1 (1970), S. $31 \mathrm{ff}$.

11 Die die Bankenverstaatlichung geschlossen unterstützten, vgl. Keesing's 1969, S. 23559.

12 Ihrer gleichzeitigen Bitte, als stellvertretender Ministerpräsident im Kabinett zu bleiben, entsprach Desai nicht, vgl. Keesing's 1969, S. 23558.

13 Text bei Keesing's 1969, S. 23558.

14 Interview in The Statesman Weekly (Calcutta) vom 14. 2. 1970, S. 16.

15 Vgl. Keesing's 1969, S. 23559, Times vom 22. 7. 1969, inzwischen ist eine gewisse Abkühlung zwischen Frau Gandhi und der Linken eingetreten, vgl. Times vom 4. 5. 1970.

16 The Banking Companies (Acquisition and Transfer of Undertakings) Ordinance 1969.

17 Central Bank of India, Bank of India, Punjab National Bank, Bank of Baroda, United Commercial Bank Canara Bank, United Bank of India, Dena Bank, Syndicate Bank, Union Bank of India, Allahabad Bank, Indian Bank, Bank of Maharashtra, Indian Overseas Bank.

18 Art. 3, 4, 5

19 Art. 10 (3). 
stellten der enteigneten Gesellschaften wurden automatisch Angestellte der neuen Banken 20. Die Gesellschafter der enteigneten Firmen sollten in Staatspapieren entschädigt werden. Berechnungsgrundlage für die Entschädigung war der Nettowert der Aktivposten nach Abzug der Verbindlichkeiten ${ }^{21}$.

Die Ordinance wurde am 21. Juli von zwei Abgeordneten der rechtsgerichteten Swatantra und Jan Sangh Parteien und einem Direktor und Gesellschafter der Central Bank of India beim Supreme Court als verfassungswidrig angegriffen ${ }^{22}$. Die Kläger bestritten die Befugnis des Präsidenten, die Ordinance nach Art. 123 ind. Verfassung zu erlassen. Diese Vorschrift gestattet dem Präsidenten gesetzesvertretende Maßnahmen, wenn beide Häuser nicht tagen und er ein sofortiges Eingreifen für notwendig hält. Die Kläger bestritten eine derartige Notwendigkeit, da die Ordinance nur 40 Stunden vor dem Beginn der neuen Sitzungsperiode des Parlaments ergangen war. Mit demselben Argument wurde das Vorgehen der Regierung im Parlament vom Präsidenten der Jan Sangh-Partei, Vajpayee, bemängelt, der sagte, wenn ein Notstand vorgelegen habe, dann nur hinsichtlich der Kongreßpartei und der Krise um die Ministerpräsidentin ${ }^{23}$. Frau Ghandi rechtfertigte sich mit dem Hinweis auf Manipulationsmöglichkeiten, wenn die Nationalisierung nicht durch eine Blitzaktion erfolgt wäre. Vor dem Supreme Court bestritt der Bundesanwalt die Justiziabilität der Entscheidung des Präsidenten über das Vorliegen der Voraussetzungen des Art. 123. Das Gericht erließ am 22. Juli eine einstweilige Anordnung, nach der die Regierung die Ordinance bis zur mündlichen Verhandlung, die auf den 11. August festgesetzt wurde, nicht vollziehen durfte ${ }^{24}$. Noch vor dieser Verhandlung wurde die Ordinance jedoch am 9. August durch ein rückwirkend vom 19. Juli geltendes Gesetz ersetzt ${ }^{25}$, das weitgehend der Ordinance entsprach. Danach wurde den enteigneten Gesellschaften ausdrücklich gestattet, andere als Bankgeschäfte zu betreiben, eine weitere Betätigung im Bankwesen wurde ihnen dagegen untersagt ${ }^{25 a}$. Daraufhin hob der Supreme Court am 11. August seine einstweilige Anordnung auf ${ }^{26}$.

\section{Die Entscheidung des Supreme Court vom 10. Februar 1970}

Das Gesetz über die Bankenverstaatlichung wurde vom Supreme Court am 10. Februar 1970 mit einer Entscheidung, die mit 10 gegen 1 Stimme erging, für unwirksam erklärt ${ }^{27}$. Die Mehrheitsentscheidung wurde von Richter Shah begründet. Das Gericht bejahte die Aktivlegitimation des Klägers, der Gesellschafter mehrerer der enteigneten Banken und Direktor der Central Bank of India ${ }^{28}$ war, mit der Begründung, die Rechte der Gesellschafter seien durch die Enteignung der Gesellschaften berührt ${ }^{29}$. Die bereits im Streit um die Wirksamkeit der Ordinance vom 19. Juli angesprochene Frage, ob deren Erlaß durch Art. 123 der Verfassung gedeckt war, ließ das Gericht dahingestellt, da sie infolge der Ersetzung dieser Rechtsnorm durch ein Parlamentsgesetz obsolet geworden war ${ }^{30}$.

20 Art. 15.

21 Art. 6 i. v. m. Schedule 2.

22 Vgl. Keesing's 1969, S. 23560.

23 Keesing's 1969, S. 23560.

24 Keesing's 1969, S. 23560.

25 Banking Companies (Acquisition and Transfer of Undertakings) Act.

25a Art. 15.

26 Keesing's 1969, S. 23561

27 Die Urteilsgründe sind ausführlich wiedergegeben in The Statesman Weekly (Calcutta) vom 14. 2. 1970, S. 7. Dort findet sich auch der Inhalt der dissenting opinion des Richters Ray.

28 Zur Person des Klägers vgl. NZZ, vom 13. 2. 1970.

29 The Statesman Weekly, a. a. O., Sp. 2.

30 A. a. O., Sp. 2. 
Die zunächst entscheidende Frage war daher die nach der Kompetenz des Gesetzgebers zum Erlaß des Gesetzes. Sie wurde vom Gericht bejaht ${ }^{31}$. Damit gab diese Entscheidung der Regierung die Möglichkeit, trotz Aufhebung des Gesetzes neue Enteignungsmaßnahmen zu ergreifen. Daß die Gesetzgebungskompetenz überhaupt bezweifelt werden konnte, liegt an der komplizierten Verteilung der Gesetzgebungszuständigkeiten auf Union und Staaten in der indischen Verfassung ${ }^{32}$. Die Union hat die ausschließliche Gesetzgebung über das Bankwesen ${ }^{33}$ und das Gesellschaftsrecht ${ }^{34}$, die Staaten über Handel innerhalb der Staaten ${ }^{35}$ und des Geldverleihs ${ }^{36}$. Konkurrierende Gesetzgebung haben Bund und Staaten über „acquisition and requisition of property“37. Das Gericht verstand unter "property“ außer Eigentum i. e. S. auch Organisation, Verbindlichkeiten, das gesamte werbende Unternehmen ${ }^{38}$. Den Einwand des Klägers, der Bundesgesetzgeber sei unzuständig, soweit Vermögen der Gesellschaft aus anderen als Bankgeschäften erfaßt werde, wurde vom Gericht zurückgewiesen, da die Existenz solcher Vermögen nicht nachgewiesen war, und sich daher auch nicht feststellen ließ, ob im Einzelfall ein Geschäftsteil erfaßt war, der in die "Staatenliste“ fiel.

Das Gesetz verletzte nach Auffassung des Gerichts jedoch Art. 19, 31(2) und 14 der indischen Verfassung. Die Ausführungen des Gerichts zu diesen Grundrechtsvorschriften verdienen eine genauere Betrachtung. Auffallend ist zunächst, daß Art. 19 und Art. 31 (2) kumulativ als Unwirksamkeitsgründe aufgeführt sind. Herrschend war in der indischen Lehre nämlich die Auffassung, daß sie sich gegenseitig ausschlössen ${ }^{39}$. Art. 19 (1) F enthält die allgemeine Eigentumsgarantie, Art. 31(2) die Enteignungsregelung. Nach herrschender, durch mehrere Gerichtsurteile bestätigter Lehre, konnte lediglich die Wirksamkeit der Enteignung nach Art. 31(2), nicht aber Art. 19 geprüft werden, sobald ein Enteignungsgesetz vorlag. Von dieser Auffassung hat sich das Gericht nunmehr ausdrücklich abgewandt ${ }^{40}$. Eigentumsverletzungen sind danach grundsätzlich sowohl an Art. 19 wie an Art. 31(2) zu überprüfen. Allerdings ist der Prüfungsmaßstab unterschiedlich. Nach Art. 19 ist nur zu prüfen, ob eine Beschränkung „reasonable“ i. S. des Art. 19(5) ist. Ist das der Fall, so kann das Gesetz lediglich noch wegen eines Verstoßes gegen die Regelung der Enteignung in Art. 31(2) unwirksam sein. Eine Beschränkung des Eigentums, die "reasonable“ ist, wurde vom Gericht bejaht, soweit den Banken ihre Banktätigkeit entzogen wird. Verneint wird sie, soweit den Gesellschaften die Möglichkeiten genommen wird, andere als Bankgeschäfte zu tätigen. Diese Fähigkeit wird ihnen zwar in Art. 15(2)E des Gesetzes ausdrücklich gelassen, aber da ihnen das gesamte Vermögen entzogen und keine Barentschädigung gewährt wird, besteht die Möglichkeit einer gewerblichen Betätigung faktisch nicht. Diese faktische Unmöglichkeit stellt das Gericht der rechtlichen gleich ${ }^{41}$ und erklärt sie für „unreasonable“, da der Zweck des Gesetzes einen Ausschluß von der Betätigung außerhalb des Bankwesens nicht erfordert.

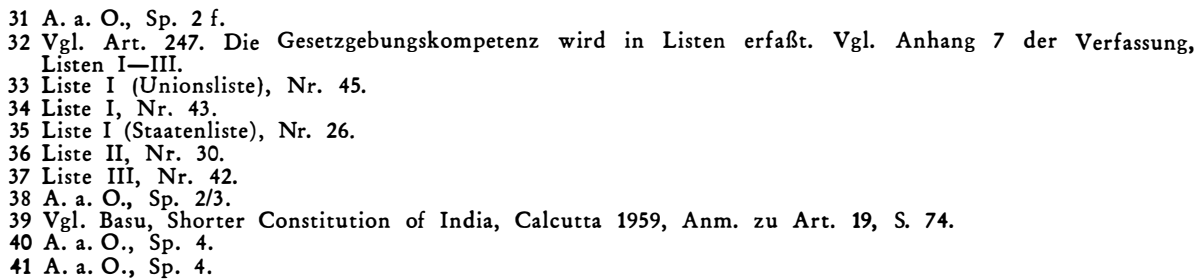


Die Unwirksamkeit des Gesetzes nach Art. 31(2) folgt demgegenüber daraus, daß eine ungenügende Entschädigung gewährt wurde ${ }^{42}$. Dieses Argument muß auf den ersten Blick überraschen, da die indische Verfassung in Art. 31(2) ausdrücklich bestimmt, daß kein Gesetz mit der Begründung angegriffen werden darf, die Entschädigung sei nicht adäquat ${ }^{43}$. Das Gericht schaffte sich die Möglichkeit, die Entschädigung trotzdem zu überprüfen, indem es die Befugnis in Anspruch nimmt, zu beurteilen, ob das Gesetz geeignete Maßstäbe für die Berechnung der Entschädigung aufstellt ${ }^{44}$. Das ist nach Ansicht des Gerichts nicht der Fall, da sich der Wert eines Unternehmens nur als Einheit bestimmen lasse, nicht als Saldo der Aktiv- und Passivposten, wie es im vorliegenden Fall geschehen war. Insbesondere waren goodwill und Zukunftserwartungen der Firmen nicht berücksichtigt und darüber hinaus Aktiva und Passiva unangemessen bewertet worden. Es erscheint zweifelhaft, wie sich diese Überlegungen mit der eindeutigen Absicht des Verfassungstextes, die Nachprüfung der Höhe der Entschädigung den Gerichten zu entziehen, in Einklang bringen läßt. Das Gericht hätte keinen Ansatzpunkt für seine Kritik gefunden, wenn die Entschädigung in festen Beträgen festgesetzt worden wäre, selbst wenn das Ergebnis für die Besitzer ungünstiger gewesen wäre. Ob tatsächlich etwas anderes gelten soll, wenn das Gesetz Berechnungsprinzipien aufstellt, die zwar nicht angemessen aber auch nicht völlig abwegig sind, ist fraglich.

Der wichtigste, zugleich aber am meisten mißverstandene Passus des Urteils ist der, in dem das Gericht dem Gesetz einen Verstoß gegen das Diskriminierungsverbot des Art. $14^{45}$ der Verfassung vorwirft. Von ausländischen Berichterstattern wurde dieser Vorwurf darauf bezogen, daß nur die 14 größten indischen Banken verstaatlicht worden waren, während die kleineren Banken und die Niederlassungen ausländischer Banken unangetastet blieben ${ }^{46}$. Eine derartige Argumentation wäre allerdings erstaunlich gewesen. Die Auswahl der übermächtigen Großbanken läßt sich ebenso sachlich rechtfertigen wie das Übergehen der ausländischen Banken, die schon aus außenpolitischen und völkerrechtlichen Rücksichten Sonderüberlegungen nötig machen. Der indische Gleichheitssatz (Art. 14) verlangt aber ebenso wie der deutsche Art. 3 Grundgesetz nur die Gleichbehandlung von Gleichem, verbietet eine sachgerechte Differenzierung also nicht ${ }^{47}$. Tatsächlich lag dem Gericht eine derartige Argumentation auch fern. Es hat die Frage, ob in der Auswahl der 14 Banken eine Diskriminierung $\mathrm{zu}$ sehen sei, zwar geprüft, aber ausdrücklich verneint, da keinerlei Anzeichen dafür vorlägen ${ }^{48}$. Die Befürchtungen ausländischer Beobachter, das Urteil zwinge die Regierung, das gesamte Bankwesen zu verstaatlichen ${ }^{49}$, war daher unbegründet. Die verbotene Diskriminierung und den Verstoß gegen Art. 14 ind. Verfassung sieht das Gericht vielmehr in einem anderen Sachverhalt, zu dessen Verständnis es wichtig ist, sich die Durchführung der Verstaatlichung $\mathrm{zu}$ vergegenwärtigen. Diese erfolgte, indem Vermögen, Geschäftsbetrieb und Belegschaft auf neu gegründete, staatliche Banken übergingen. Die enteigneten Gesellschaften blieben also, wenn auch ohne Vermögen und Geschäftsbetrieb, bestehen. Ihnen wurde jedoch verboten, Bankgeschäfte zu tätigen ${ }^{50}$.

42 A. a. O., Sp. 5.

43 Vgl. dazu Basu, a. a. O., Anm. zu Art. 31 (2), S. 140.

44 . . a method relevant to the determination of compensation.

45 The State shall not deny to any person equality before the law or the equal protection of the laws within the territory of India.

$46 \mathrm{NZZ}$ vom 13. 2. 1970, Le Monde vom 11. 2. 1970, Times vom 11. 2. 1970.

$47 \mathrm{Vgl}$. Basu, a. a. O., Anm. zu Art. 14, S. $24 \mathrm{ff}$.

48 A. a. O., Sp. 4.

49 NZZ vom 13, 2. 1970, Times vom 11, 2. 1970.

$50 \mathrm{Vgl}$. Art. 15 des Gesetzes. 
In diesem Verbot sieht das Gericht einen Verstoß gegen das Diskriminierungsverbot des Art. 14, da gleichzeitig andere Privatbanken tätig bleiben durften und sogar neue Banken gegründet werden konnten. Bedenkt man, daß die enteigneten Gesellschaften wegen ihrer Größe ausgesucht wurden, daß sie aber nach der Entziehung ihres Vermögens und ihres Geschäftsbetriebes allenfalls in der Größenordnung Bankgeschäfte hätten betreiben können, wie die nicht enteigneten kleineren Banken, erscheint diese Argumentation nicht ganz abwegig. Allerdings berücksichtigt das Gericht wohl zuwenig, daß die enteigneten Gesellschaften auf Grund ihres goodwill und ihrer Beziehungen zu den wichtigsten Wirtschaftskreisen sehr schnell zu einer Konkurrenz für die neuen Staatsbanken werden könnten.

\section{Die Renationalisierung}

Unmittelbar nachdem das Urteil verkündet worden war, gingen die Regierungsjuristen an die Arbeit, um eine neue - diesmal unangreifbare - Rechtsgrundlage für die Bankenverstaatlichung zu schaffen ${ }^{51}$. Das bereitete angesichts der sorgfältigen Begründung des Urteils und der deutlichen Hinweise, die es enthielt, keine Schwierigkeit.

Die Aufgabe der Regierung war nicht juristisch, sondern politisch. Das Urteil ließ sieht man von dem radikalen Vorstoß zur Abschaffung der Eigentumsgarantie aus der Reihe der Anhänger der Ministerpräsidentin ${ }^{52}$ einmal ab - zwei Wege zu einer rechtlich unanfechtbaren Enteignung der Banken: der „linke“ Weg war die Enteignung des gesamten Bankwesens, der "rechte“ die Erlaubnis zur weiteren Betätigung im Bankgeschäft für die enteigneten Gesellschaften. Trotz starken Drucks linker Kräfte ${ }^{53}$ wählte die Ministerpräsidentin den zweiten Weg. Durch eine - inzwischen durch ein Gesetz ersetzte ${ }^{53 a}$ - Ordinance des Präsidenten wurden die 14 Banken erneut enteignet ${ }^{54}$. Das vom Gericht als diskriminierend angesehene Verbot der Betätigung im Bankwesen fiel fort. Die Entschädigung wurde höher als zuvor, vor allem aber diesmal in absoluten Größen festgesetzt, so daß eine Überprüfung der Bewertungsmaßstäbe nicht mehr möglich ist. Schließlich wird die Entschädigung nunmehr nach Wahl der Betroffenen auch bar geleistet, und zwar an die Gesellschafter selbst, so daß sie mit diesem Geld geschäftlich tätig sein können. Damit entfällt der Vorwurf, den Gesellschaften werde auch die weitere wirtschaftliche Betätigung de facto unmöglich gemacht.

Damit sind alle Mängel, die das Gericht an dem früheren Gesetz festgestellt hatte, beseitigt. Die Wahl der neuen Enteignungsmaßnahme hat für die Ministerpräsidentin einen doppelten Vorteil: indem sie die Banken innerhalb weniger Tage erneut verstaatlichte, machte sie deutlich, daß sie an ihrer sozialistischen Wirtschaftspolitik festzuhalten gedenkt. Da sie andererseits dem Druck, nunmehr alle Banken einschließlich der ausländischen zu verstaatlichen, nicht nachgab, konnte sie ihre Aussage vom 19. Juli 1969 glaubhaft machen, die Bankenverstaatlichung bedeute keinen Beginn einer Aera der Nationalisierungen ${ }^{55}$. Das dürfte sich zugunsten des Investitionsklimas gerade im Hinblick auf das Ausland vorteilhaft auswirken.

51 Vgl. The Statesman Weekly vom 14. 2. 1970, S. 1, Times vom 11. 2. 1970.

52 Vgl. Times vom 20. 2. 1970.

$53 \mathrm{Vgl}$. The Statesman Weekly vom 14. 2. 1970, S. 1

53a Keesing's 1970, S. 23956

54 Vgl. Zum Inhalt The Statesman Weekly vom 21. 2. 1970, S. 6, The Overseas Hindustan Times vom 21. 2. 1970 , S. 16. NZZ vom 16. 2. 1970, Keesing‘s 1970, S. 23956.

55 Vgl. Keesing's 1969, S. 23559, NZZ vom 21. 7. 1969. 